\title{
Roche bets on alpha-synuclein for Parkinson's
}

In December, Roche forged a deal with Elan spinoff Prothena, worth up to $\$ 600$ million, to develop and commercialize a monoclonal antibody to treat Parkinson's disease. The experimental drug, PRX002, targets alphasynuclein, a protein known to aggregate in the brains of individuals with Parkinson's disease and other neurodegenerative disorders, such as dementia with Lewy bodies. "I am a big supporter of alpha-synuclein as a target and also of the use of antibodies," says Christopher Dobson, a chemist at the University of Cambridge, UK, who studies the relationship between protein misfolding and disease. Other companies are tackling alpha-synuclein with active immunization and small molecules for Parkinson's disease and other related neurodegenerative conditions. Although all are at early stages of development, Roche's recent deal signals mounting interest in this target.

"Under given conditions, the extent of alpha-synuclein aggregation is dominated by the rates of key steps in the series of reactions involved in the self-assembly process," says Dobson (Cell 149, 1048-1059, 2012). An antibody that binds to alpha-synuclein may slow down one or more these reactions and inhibit the protein's ability to form aggregates, he explains. Antibody binding might also stimulate clearance of alpha-synuclein, reducing the probability that aggregates will form, Dobson adds.

PRX002 is a humanized monoclonal antibody that binds to the C-terminus of alphasynuclein protein. The partners will take the experimental drug into phase 1 trials, in an agreement that includes using Roche's proprietary Brain Shuttle delivery technology to boost delivery of the antibodies into the brain. As part of the PRX002 deal, the Basel-based Roche will pay Prothena \$45 million upfront. The Dublin-based biotech is also eligible to receive up to $\$ 380$ million in development, regulatory and first commercial sales milestones, plus up to an additional \$175 million in commercial milestones on sales outside of the US. In the US, Roche and Prothena will share development and commercialization costs in a ratio of $70 \%$ and $30 \%$, respectively.

Other drugs in development that target alpha-synuclein to treat Parkinson's disease include Vienna-based AFFiRiS' PD01A antibody vaccine, which uses the company's proprietary AFFITOME technology to generate short peptides that mimic alpha-synuclein to elicit an immune response, presently in phase 1 clinical trials, and Kirkland,

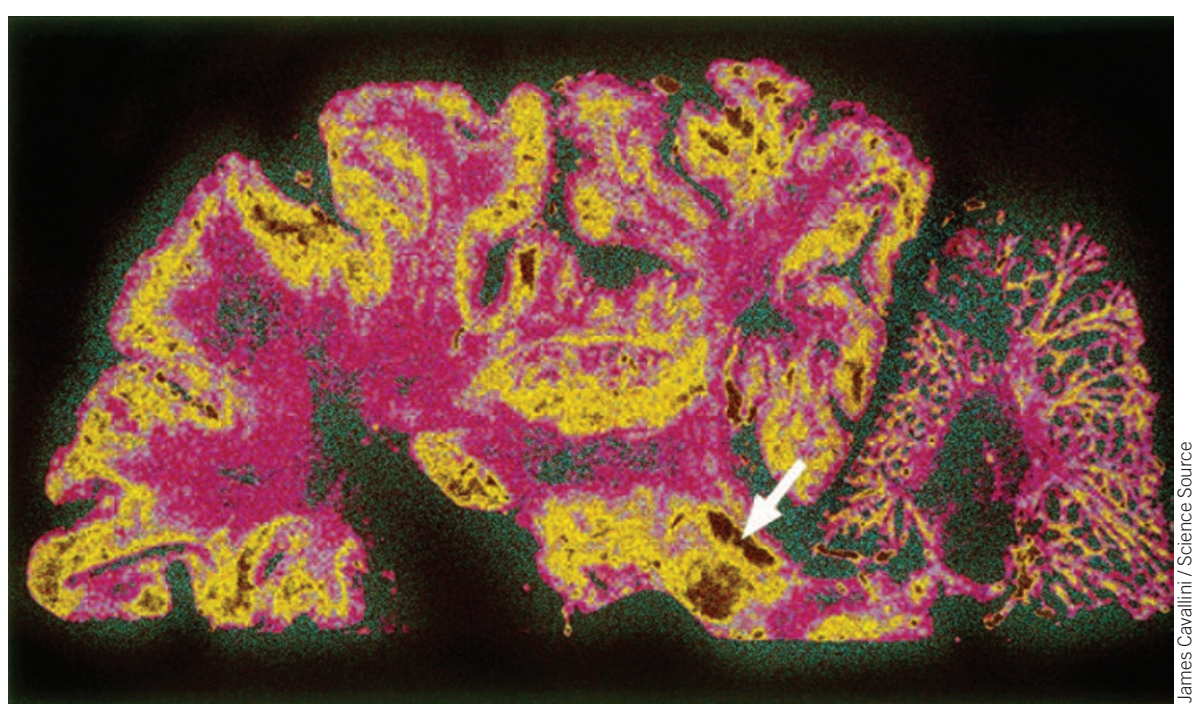

Coronal section through the brain showing the insoluble aggregates that characterize Parkinson's disease.
Washington-based ProteoTech's Synuclere, a small-molecule compound in preclinical stages.

Small molecules in development work similarly to antibodies. ProteoTech's Synuclere, for example, binds to alphasynuclein monomers to prevent aggregation, but also binds to aggregates, reducing the synuclein fibrils into amorphous nonaggregating structures that can be cleared out of the brain by microglia, says Alan Snow of ProteoTech. Small molecules carry the advantage that they are cheaper to develop and manufacture and tend to be safe when taken over long time periods, as would be necessary to treat Parkinson's disease, says Dobson. They are also generally better at penetrating the blood-brain barrier. Antibody drugs, however, tend to be more specific to their targets and bind very tightly, Dobson adds. Also, because the rate of alpha-synuclein aggregate formation is dependent on concentration, “you don't need to perturb a large number of synuclein molecules to reduce the probability of aggregates forming," Dobson says.

Aside from direct targeting, companies are using other strategies to reduce alphasynuclein to treat Parkinson's disease. Biogen Idec of Cambridge, Massachusetts, recently announced it would team up with Amicus Therapeutics of Cranbury, New Jersey, to develop small-molecule drugs that increase the activity of the lysosomal enzyme glucocerebrosidase. A deficiency of this enzyme can lead to the accumulation of alpha-synuclein in the brain.

Gunjan Sinha Berlin

\section{Corrections}

In the June 2013 news brief, DuPont pays $\$ 1.75$ for Monsanto next-gen traits (Nat. Biotechnol. 31, $477,2013)$, the amount of money in the title was given as " $\$ 1.75$ " instead of "1.75B." The error has been corrected in the HTML and PDF versions of the article.

In the news analysis "From the bottom of a DNA cocktail glass" (Nat. Biotechnol. 31, 868, 2013), Natalie Moll's previous affiliation was incorrectly given as former head of Assobiotec. She is former head of public affairs for Assobiotec. The error has been corrected in the HTML and PDF versions of the article.

In the news analysis "J\&J courts biotech in clusters" (Nat. Biotechnol. 31, 769-770, 2013), Rodin Therapeutics was listed as Robin Therapeutics, and the California Institute for Quantitative Biosciences was incorrectly called Quantitative Biosciences 3. The errors have been corrected in the HTML and PDF versions of the article.

In the news analysis "Social networks attempt to spark academic-university collaborations" (Nat. Biotechnol. 30, 901-903, 2013), the title should have read "academic-industry collaborations." The error has been corrected in the HTML and PDF versions of the article. 